\title{
Dynamics and responses to mortality rates of competing predators undergoing predator-prey cycles
}

\author{
Peter A. Abrams, ${ }^{\mathrm{a}, *}$ Chad E. Brassil, ${ }^{\mathrm{a}}$ and Robert D. Holt ${ }^{\mathrm{b}}$

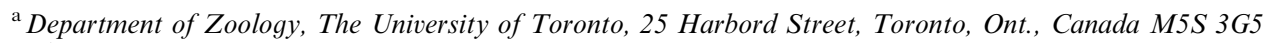 \\ ${ }^{\mathrm{b}}$ Department of Zoology, University of Florida, P.O. Box 118525, Gainesville, FL 32611-8525, USA
}

Received 30 April 2002

\begin{abstract}
Two or more competing predators can coexist using a single homogeneous prey species if the system containing all three undergoes internally generated fluctuations in density. However, the dynamics of species that coexist via this mechanism have not been extensively explored. Here, we examine both the nature of the dynamics and the responses of the mean densities of each predator to mortality imposed upon it or its competitor. The analysis of dynamics uncovers several previously undescribed behaviors for this model, including chaotic fluctuations, and long-term transients that differ significantly from the ultimate patterns of fluctuations. The limiting dynamics of the system can be loosely classified as synchronous cycles, asynchronous cycles, and chaotic dynamics. Synchronous cycles are simple limit cycles with highly positively correlated densities of the two predator species. Asynchronous cycles are limit cycles, frequently of complex form, including a significant period during which prey density is nearly constant while one predator gradually, monotonically replaces the other. Chaotic dynamics are aperiodic and generally have intermediate correlations between predator densities. Continuous changes in density-independent mortality rates often lead to abrupt transitions in mean population sizes, and increases in the mortality rate of one predator may decrease the population size of the competing predator. Similarly, increases in the immigration rate of one predator may decrease its own density and increase the density of the other predator. Proportional changes in one predator's birth and death rate functions can have significant effects on the dynamics and mean densities of both predator species. All of these responses to environmental change differ from those observed when competitors coexist stably as the result of resource (prey) partitioning. The patterns described here occur in many other competition models in which there are cycles and differences in the linearity of the responses of consumers to their resources.
\end{abstract}

(C) 2003 Elsevier Science (USA) All rights reserved.

\section{Introduction}

Much current thought on competition is based upon the Lotka-Volterra model of two competing species. Because this model always has a stable equilibrium when two species coexist, the role of population cycles is seldom considered in empirical studies when trying to understand the interactions between coexisting competitors. The Lotka-Volterra model also leads to expectations about the responses of the densities of each competitor to parameters affecting the fitness of the other species, such as its mortality rate. For instance, continuous increase in the per capita mortality rate of

${ }^{*}$ Corresponding author. Fax: +416-978-8532.

E-mail addresses: abrams@zoo.utoronto.ca (P.A. Abrams), brassil@zoo.utoronto.ca (C.E. Brassil), predator@zoo.ufl.edu (R.D. Holt). one species decreases its equilibrium density and increases the equilibrium density of its competitor. Similar qualitative behaviors characterize many models of direct competition in which there is stable coexistence. Similarly, increasing the immigration rate of that species will increase its density and decrease that of its competitor. Because empirical studies of competitor and community structure are guided by theoretical expectations, it is important to identify situations in which these expectations are unlikely to be realized. In this paper, we show that models with explicit resource dynamics, differences in the linearity of the predators' responses, and the potential for sustained oscillations, can exhibit sharply different effects. Because it has long been known that consumer-resource interactions can easily produce cycles (Rosenzweig and MacArthur, 1963), and that cycles can alter conditions for coexistence (Armstrong and McGehee, 1980), it is surprising 
that the implications of cycling for interactions between competitors have not received more attention.

In a previous article (Abrams and Holt, 2002), we argued that a mechanism of coexistence proposed by Koch (1974) and by Armstrong and McGehee (1976a, b, 1980), McGehee and Armstrong (1977) may play an important role in species coexistence in natural communities. This mechanism is based on differences in the nonlinearity of two competitors' responses to a single resource combined with consumer-resource cycles generated by the interaction of one of those consumers with the resource. The more linear species has a relatively high resource requirement, and therefore cannot exclude the species with the more nonlinear response. The latter generates cycles when alone with the resource; these cycles increase the mean resource density so that linear species can invade. If the 'robustness' of coexistence is measured by the range of prey (resource) requirements for zero population growth (i.e., efficiencies) that allow coexistence, then Armstrong and McGehee's model can produce very robust coexistence. The range of efficiencies allowing coexistence via Armstrong and McGehee's mechanism is often as wide as the range of efficiencies allowing coexistence of competing predators which do not cycle, but which have a high degree of partitioning of prey species (Abrams and Holt, 2002). This observation, together with the fact that a significant number of species cycle (Ellner and Turchin, 1995; Kendall et al., 1998), suggests that cases of coexistence via the Armstrong-McGehee mechanism may be reasonably common. However, well-supported empirical examples of coexistence due to competitor-resource cycles are absent from the published literature. By describing the dynamics of such competitive systems, and how they respond to changes in mortality rates, we hope to find dynamical signals that may help identify examples in natural systems. Moreover, Armstrong and McGehee's (1980) model exhibits a wider range of dynamics than has been described previously, including chaos, alternative limit cycles, and long-lasting transients.

\section{The model}

We begin by analyzing the model that Armstrong and McGehee (1980) used to illustrate nonequilibrial coexistence of two species on a single biotic resource. This system is characterized by logistic prey growth, a linear functional response for one predator, and a type- 2 functional response for the other predator. We argue below that this model displays many dynamics that are common to a much broader set of models characterized by differences in the linearity consumer species and endogenously generated cycles. The dynamics of the two predators (consumers), $P_{1}$ and $P_{2}$, and the prey (resource), $N$, are given by

$$
\begin{aligned}
& \frac{d P_{1}}{d t}=P_{1}\left(\frac{B_{1} C_{1} N}{1+h C_{1} N}-D_{1}\right), \\
& \frac{d P_{2}}{d t}=P_{2}\left(B_{2} C_{2} N-D_{2}\right), \\
& \frac{d N}{d t}=r N\left(1-\frac{N}{K}\right)-\frac{C_{1} N P_{1}}{1+h C_{1} N}-C_{2} N P_{2} .
\end{aligned}
$$

The parameter $B_{i}$ is the conversion efficiency of food into offspring for predator $i, h$ is the handling time per prey item for predator $1, D_{i}$ is a density independent death rate, $C_{i}$ is a searching predator's attack rate, and $r$ and $K$ are logistic growth parameters. The variables in this equation can be scaled to reduce the number of parameters, as follows: $t^{\prime}=r t ; N^{\prime}=N / K$; and $P_{1}{ }^{\prime}=$ $P_{1} /\left(K B_{1}\right)$, and $P_{2}{ }^{\prime}=P_{2} /\left(K B_{2}\right)$. After making substitutions and dropping the primes on the new variables, the model becomes

$$
\begin{aligned}
& \frac{d P_{1}}{d t}=P_{1}\left(\frac{a_{1} N}{1+b N}-d_{1}\right), \\
& \frac{d P_{2}}{d t}=P_{2}\left(a_{2} N-d_{2}\right), \\
& \frac{d N}{d t}=N(1-N)-\frac{a_{1} N P_{1}}{1+b N}-a_{2} N P_{2},
\end{aligned}
$$

where the new parameters are $a_{1}=K B_{1} C_{1} / r, a_{2}=$ $K B_{2} C_{2} / r, b=K C_{1} h, d_{1}=D_{1} / r, d_{2}=D_{2} / r$. Below we refer to predator 1 as the nonlinear predator, and predator 2 as the linear predator.

There is no equilibrium point with positive densities of all three species. However, the species can coexist for some range of the parameters $a_{2}$ and $d_{2}$ if $P_{1}$ and $N$ undergo limit cycles in the absence of the second predator (McGehee and Armstrong, 1977; Armstrong and McGehee, 1980). Limit cycles occur in the subsystem lacking predator 2 if

$d_{1}<\frac{a_{1}(b-1)}{b(b+1)}$.

The right-hand side of this inequality will be referred to as the stability threshold value of $d_{1}$. Here we only consider cases where $d_{1}, a_{1}$ and $b$ satisfy (3). We consider coexistence to occur if each predator species can increase when it is rare and the other predator and prey are undergoing their limiting dynamics (stable point or limit cycle). This differs from Armstrong and McGehee's (1980) definition of coexistence, and there are circumstances when such 'mutual invasion' definitions of coexistence may be misleading (Armstrong and McGehee, 1980). The most serious problem is the possibility that initial invasion is eventually followed by exclusion of the invading species. This outcome was never observed in any numerical solution of Eq. (2), or in any other model that we consider here. Previous examples of this phenomenon (the reversal of initially successful invasion) have had alternative attractors for the subsystem 
being invaded (Abrams and Shen, 1989; Case, 1995), unlike the models considered here. A second potential problem with the mutual invasion definition of coexistence is that invasion from very low densities may be impossible, yet an attractor with bounded positive densities of all species may be attainable, given a sufficiently high initial density. This was also not observed in our simulations. Were such outcomes to occur, maintenance of all three species in a stochastic environment would be unlikely, as each predator would probably experience low enough densities to lead to eventual extinction.

\subsection{Numerical methods}

Given our mutual invasion definition, two conditions must be met for coexistence. First, predator species 1 must be able to invade the system with predator 2 and the prey at equilibrium. This subsystem has a globally stable equilibrium; the invasion condition is $d_{2} / a_{2}>d_{1} /\left(a_{1}-b d_{1}\right)$. Secondly, predator species 2 must be able to invade the system in which predator 1 and the prey are undergoing limit cycle dynamics. Invasion of predator 2 is possible if $d_{2} / a_{2}\left\langle\langle N\rangle_{1}\right.$, where $\langle N\rangle_{1}$ denotes the average prey density over a limit cycle. This condition follows from the requirement that the time average of the per capita growth rate of predator 2 must be positive when it is too rare to influence resource levels.

In the numerical results below, we include a small immigration rate of prey. This prevents unrealistically low prey densities, and is biologically plausible because prey are usually more widely distributed than are their specialist predators. Prey immigration leads to a term, $I$, being added to the right-hand side of Eq. (1c), and a scaled term, $i(=I /(K r))$ being added to Eq. (2c). If $i$ is several orders of magnitude less than one, there are only small effects on equilibrium predator densities and on the stability condition given by Eq. (3), but the prey is buffered from excursions to very low densities. In the simulations of Eq. (2) reported below, we generally assumed $i=0.0001$; minimum prey densities are typically no less than $10^{-4}$ times the carrying capacity.

Previous work (Abrams and Holt, 2002) shows that coexistence is only possible over a wide range of death rates (or resource requirements) for each competing predator if the parameter $b$ in Eq. (2) is much greater than unity. Here we generally assume that $b=10$, which is on the order of that observed in several experimental studies (Abrams et al., 1990; Gross et al., 1993; Messier, 1994; Eby et al., 1995; Ruesink, 1997). A more limited set of simulations was carried out for smaller $b(=1.25$, 3 , or 5 ). Given that $b=10$, the nature of the population cycles produced by species 1 in the absence of species 2 is determined by the parameters $a_{1}$ and $d_{1}$. In our most extensive simulations, we examined system dynamics for a range of $a_{1}$ values spanning the prey's intrinsic growth rate $\left\{a_{1}=0.2, a_{1}=1, a_{1}=5\right\}$. For each $a_{1}$, we examined death rates, $d_{1}$, approximately $20 \%$ or $60 \%$ below the stability threshold set by inequality (3); i.e. $d_{1}=0.06545 a_{1}$, and $0.03273 a_{1}$. For each of these 6 parameter combinations, we numerically determined the dynamics produced over a wide grid of $a_{2}$ and $d_{2}$ values that allow both predator species to coexist. The results from this initial set of simulations were used then to explore in more detail areas of parameter space in which a variety of different dynamic behaviors were observed. This included much larger and much smaller values of the parameters, $a_{i}$, and values of $d_{1}$ both closer to and further below the stability threshold value.

Numerical integrations were carried out using a fourth order Runge-Kutta procedure with adaptive step size (Press et al., 1992) coded in C. All numerical integrations were run for at least 20,000 time units to attain the limiting dynamics. Many results were checked using the function NDSolve in Mathematica 4.0 (Wolfram Research, 1999) with a setting of infinity for the AccuracyGoal. Lyapunov exponents were calculated using a routine based on Wolf et al. (1985) and coded in $\mathrm{C}$; these results were checked using the program Dynamics II (Nusse and Yorke, 1998). Lyapunov exponents were estimated over an additional 20,000 time units.

\subsection{Dynamical patterns}

The limiting dynamics observed across the entire set of simulations can be roughly categorized into three main types (see Fig. 1), which we refer to as 'synchronous cycles', 'asynchronous cycles', and 'chaotic dynamics'. A description of these three types of dynamic behavior and a rough description of the parameter values yielding each type is as follows:

\subsubsection{Synchronous cycles}

The most commonly observed population dynamics were simple limit cycles of relatively short period, with the two predator populations varying in a roughly synchronous fashion (as in Fig. 1A). In comparison with the limit cycle dynamics of the unstable predator interacting alone with the prey, the amplitudes of cycles in the full system are smaller and the periods shorter. Larger cycles benefit the linear predator relative to the nonlinear, but increases in the abundance of the linear predator reduce cycle amplitude. In most cases of synchronous cycles, the correlation coefficient of the two predator densities over time was between 0.8 and 1.0. The limit cycles are usually simple in the sense that all local maxima (minima) of a given variable are identical. However, some synchronous cycles with more complex structure were observed over limited parameter ranges. Synchronous limit cycles were the predominant 
Densities $\left(\mathrm{N}_{1}\right.$ thick, $\mathrm{N}_{2}$ thin, $\mathrm{R}$ dashed)

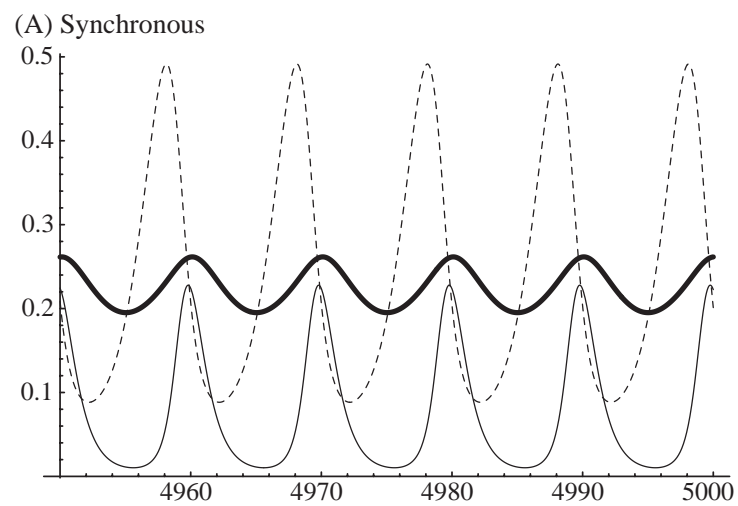

(B) Asynchronous

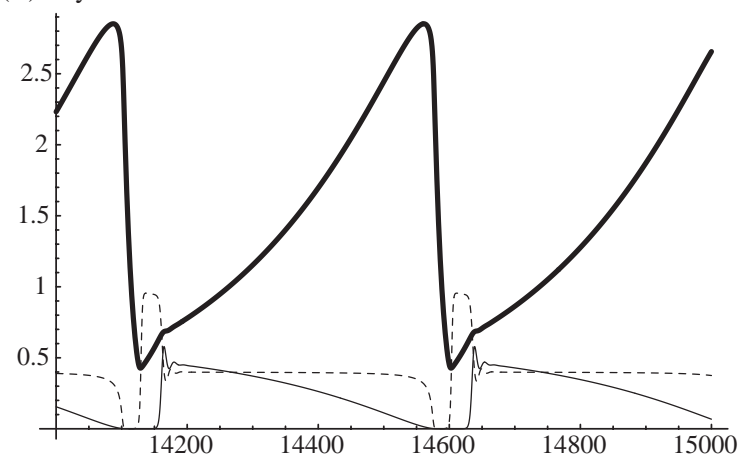

(C) Chaotic

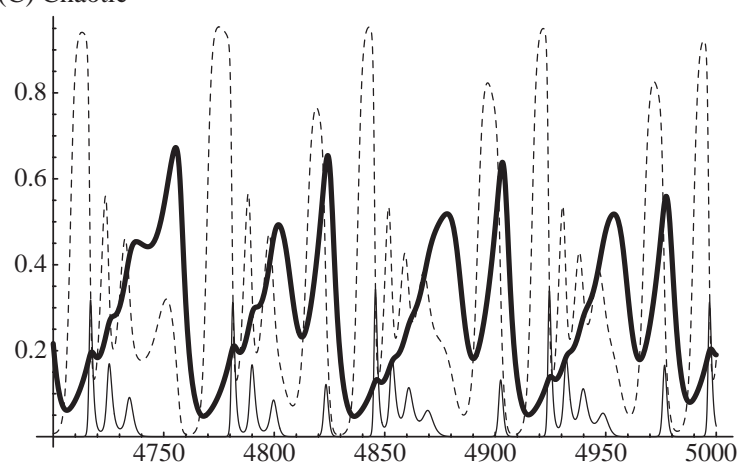

Time

nous cycles occurred over the entire range of $d_{2}$ permitting coexistence when $d_{1}$ was less than $1 / 2$ of its stability threshold value $\left(d_{1}<0.2045\right)$. Synchronous cycles also represent the only type of dynamics observed when $b$ is close to 1 or when both $d_{2}$ and $a_{2}$ are sufficiently small relative to 1 , even if $d_{1} / a_{1}$ is near the stability threshold. One interpretation of the Armstrong-McGehee model is that it involves 'temporal niche partitioning', in which each species enjoys a competitive advantage during some time period. This is true, but our results show that such niche partitioning does not imply temporal displacement in peaks and troughs in abundance.

\subsubsection{Asynchronous cycles}

In these relatively long-period cycles, there are phases of nearly constant prey densities and opposite, nearly monotonic, changes in the densities of the two predator species (decline in the linear, and increase in the nonlinear predator) during a significant fraction of the cycle period. These periods of gradual replacement of the linear predator are characterized by prey densities slightly below the replacement level required by the linear predator, and are followed by brief periods of cycling accompanied by rapid shifts in the relative abundances of the two predators. These reversals occur when the linear species becomes so rare it can no longer prevent cycles driven by the nonlinear predator. Fig. 1B is an example of this type of cycle. Asynchronous cycles are often complex in structure, having several different local maxima in density of each variable over the timecourse of a single cycle. The correlation coefficient between the densities of the two predators undergoing asynchronous cycles is usually negative; if positive, it is generally less than 0.3 . However, the dividing line between this type of cycle and the synchronous ones is to some extent arbitrary, because the fraction of the cycle during which prey density was relatively constant varied considerably, as did the constancy of the prey. For later figures, we adopt a somewhat arbitrary criterion of a negative correlation coefficient of predator densities for distinguishing asynchronous from synchronous cycles. Asynchronous cycles were not observed when the value of the parameter $b$ was close to 1 , the minimum $b$ for which cycles are possible. The range of other parameters producing asynchronous cycles increases as $b$ increases. Given that $b$ is large, asynchronous cycles having very long periods are often observed for a narrow range of death rates, $d_{2}$, near the lower boundary of the range of $d_{2}$ allowing coexistence. In this case, the two predators are very nearly equal in their prey requirements. Asynchronous cycles with shorter periods are observed for other, broader ranges of parameters. Asynchronous cycles appeared to occur over the broadest ranges of parameters when the attack rate of the nonlinear predator, $a_{1}$, was on the order of 1

type of dynamics observed over the vast majority of $\left\{d_{2}, a_{2}\right\}$ parameter space permitting coexistence when the value of $d_{1} / a_{1}$ was significantly (i.e., on the order of $50 \%$ or more) below the stability threshold set by condition (3). For example, when $a_{1}=a_{2}=5$, synchro- 
or less, given that $b$ was large relative to 1 . When these conditions on $a_{1}$ and $b$ were satisfied, asynchronous cycles were most common when $d_{1}$ was relatively close to its stability threshold value. These conditions allow the linear predator to maintain stability of the resource population for significant periods of time as its own population gradually declines, and the population of the nonlinear predator increases; eventually, the inherent instability of the latter predominates and so in effect, the system shifts between alternative dynamical domains.

\subsubsection{Chaotic dynamics}

Chaotic dynamics (identified by a positive value of the largest of the three Lyapunov exponents) produced more ambiguous correlations between the densities of the two species. Chaotic dynamics occurred over a substantial fraction of parameter space when: (i) the value of $b$ in Eq. (2) was relatively large; (ii) the value of $d_{1}$ in Eq. (2) was moderately close to the stability threshold given by Eq. (3); (iii) the scaled attack rate $\left(a_{1}\right)$ of the nonlinear predator was significantly greater than unity; and (iv) the prey requirement for zero growth of species $2, d_{2} / a_{2}\left(=N_{2} *\right)$, was intermediate in the range of values allowing coexistence. Chaos was rare or absent if the demographic rates of the linear predator were sufficiently slow relative to the prey $\left(d_{2}, a_{2} \ll 1\right)$. When $a_{1}=a_{2}=5$, chaotic dynamics were only observed when $d_{1}$ was greater than approximately $1 / 2$ of its stability threshold value $\left(d_{1}=0.40909\right)$. For these values of $a_{i}$ with $b=10$, chaos was absent for $d_{1} \leqslant 0.21$, and the broadest range of $d_{2}$ values produced chaos when $d_{1}$ was between approximately 0.28 and 0.38 . Fig. $1 \mathrm{C}$ is an example of chaotic dynamics in this system when $d_{1}=$ 0.3273 and $d_{2}=1.7$.

Most of the above results can be understood by considering the ability of the linear competitor to control the dynamics of the resource while the nonlinear species increases to a density where it is able to drive cycles of the prey. Such phases are a key characteristic of asynchronous cycles. Small demographic rates of the nonlinear species and a value of $d_{1}$ near the stability threshold both reduce the strength of the destabilizing effect of the nonlinear species, while large demographic rates of the linear species increase its ability to temporarily stabilize resource densities. These conditions lead to asynchronous cycles or chaos. The opposite conditions increase the relative impact of the nonlinear species on resource dynamics, and tend to lead to synchronized cycles. Nonsynchronous dynamics require a large half saturation constant (large $b$ ); this largely decouples the demographic rates of the nonlinear species from resource density over a wide range of resource densities. In this case, damped cycles driven by the linear predator and the prey do not entrain cycles in the nonlinear predator, because the latter experiences little variation in its per capita growth rate when the resource fluctuations are modest.

The above classification of dynamics is neither complete nor unambiguous. There are a variety of intermediate cases; for example, chaotic dynamics with a very small positive maximum Lyapunov exponent arise that appear superficially similar to the asynchronous cycles in Fig. 1B. Another example is roughly asynchronous cycles in which the phase of nearly constant prey density is replaced by one in which prey density fluctuates above and below the prey requirements of the linear predator. Fig. 2 (see next paragraph) illustrates another type of dynamics observed for a narrow range of parameter values. Nevertheless, most of the observed dynamics could be clearly assigned to one of the above three categories.

Alternative attractors were found for small regions of parameter space. In all cases observed, the two alternatives were a synchronous limit cycle of small amplitude and an asynchronous cycle with much larger amplitude fluctuations in densities and a much longer period. For some parameter sets, asynchronous cycles were also observed as long-lasting transients for a range of parameters in which the ultimate attractor was a simple synchronous cycle. Such cases were characterized by having all Lyapunov exponents very close to zero. This occurred for parameters where the linear species had a death rate close to the minimum value that would allow it to coexist with the nonlinear species. In these cases, dynamics similar to those shown in Fig. 1B could persist for thousands of time units, but would eventually resolve into the pattern shown in Fig. 1A. An example of an intermediate stage in such a transition is given in Fig 2. In this transition period between the two types of dynamics, there is a slow long-period cycle with negatively correlated densities of the two predators that

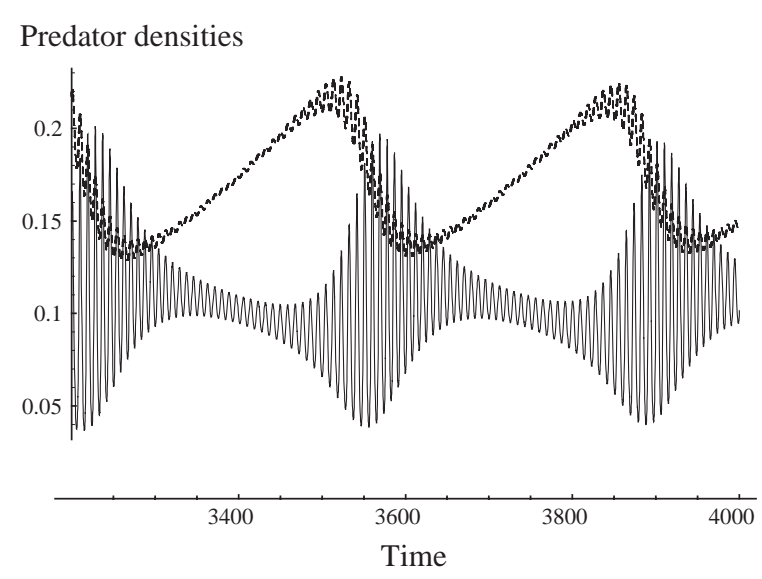

Fig. 2. An intermediate stage in the transition between a long-lasting but transient asynchronous cycle to a synchronous cycle. Parameter values are: $a_{1}=a_{2}=5 ; d_{1}=0.327273 ; d_{2}=0.97 ; i=0.0001 ; b=10$. The density of predator 1 is given by the upper (dashed) line, and predator 2 by the lower (solid) line. 
(A) $a_{l}=5, d_{l}=0.38$

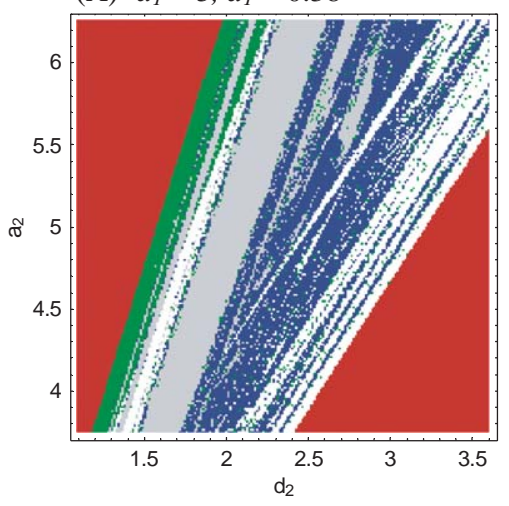

(C) $a_{l}=1, d_{l}=0.076$

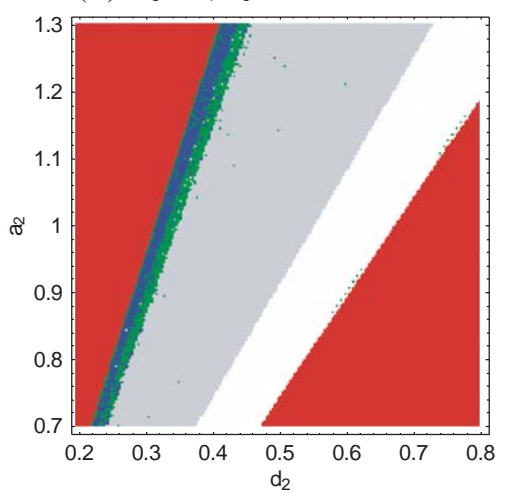

(B) $a_{l}=5, d_{1}=0.327273$

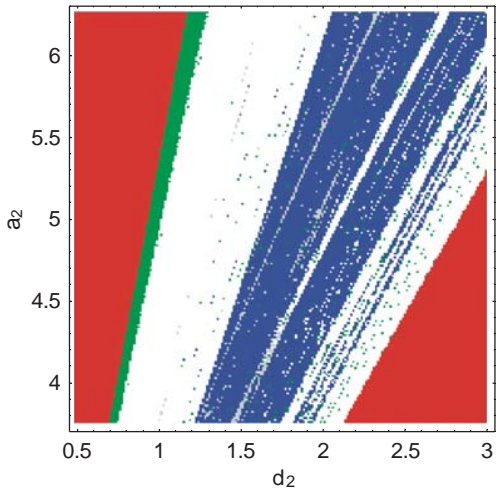

(D) $a_{l}=1, d_{l}=0.0654545$

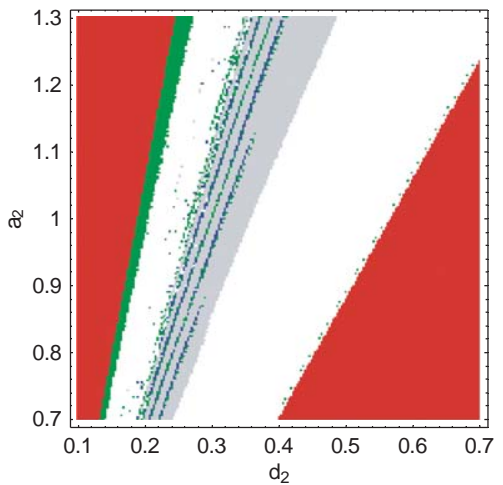

(E) $a_{1}=5, d_{1}=0.327273$

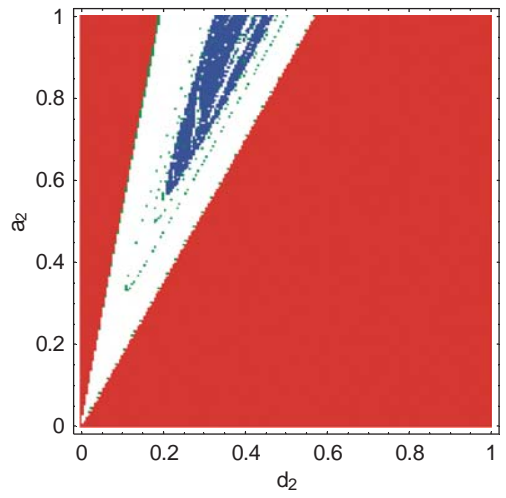

Fig. 3. Dynamics across a grid of $\left\{a_{2}, d_{2}\right\}$ values for different combinations of $a_{1}$ and $d_{1}$. The values of $a_{1}$ and $d_{1}$ are given on top of each panel. The value of $b$ is 10 in all cases. The color-coding of dynamical behaviors is: red-exclusion; blue-chaotic; green-indeterminate (absolute value of all Lyapunov exponents less than 0.001); white-synchronous cycles; gray-asynchronous cycles. Cycles were classified as synchronous if the correlation coefficient of predator densities was greater than zero, and asynchronous if the correlation coefficient was less than zero. All simulations used a common set of initial densities.

is overlain upon a rapid, smaller-amplitude cycle with positively correlated densities. There are some narrow parameter ranges where the attractor of the system had dynamics similar to those shown in Fig. 2.

Fig. 3 illustrates typical patterns of occurrence of different dynamics over continuous ranges of the parameters $a_{2}$ and $d_{2}$. Given that we restricted the parameter $b$ to large values ( $b=10$ in most simulations), the type of dynamics depended on the four remaining parameters, $a_{1}, a_{2}, d_{1}$, and $d_{2}$. Figs. $3 \mathrm{~A}$ and $\mathrm{B}$ show the range of dynamics that occur over a grid of potential $a_{2}$ and $d_{2}$ values for cases in which $a_{1}$ is significantly greater than $1\left(a_{1}=5\right)$. This grid is shown for two values of $d_{1}$ that are approximately 0.93 and 0.80 times the stability threshold. Dynamics are classified into the categories identified above. We have used a conservative method of classifying cycles as asynchronous cycles based on a periodic attractor with a negative correlation coefficient between the densities of the two predators. In both Figs. $3 \mathrm{~A}$ and $\mathrm{B}$, a broad intermediate range of $d_{2}$ 
produces either asynchronous cycles or chaotic dynamics. However, the relative sizes and locations of parameter values producing asynchronous cycles are quite different in the two figures, with asynchronous cycles being more common when $d_{1}$ is larger. In both figures, there is a narrow range of $d_{2}$ close to the minimum that will allow coexistence, where very longperiod asynchronous cycles occur; these generally have all Lyapunov exponents very close to zero. The effect of lower values of the predator attack rates is illustrated by Figs. $3 \mathrm{C}$ and D, in which $a_{1}=a_{2}=1$. In Figs. 3C and $\mathrm{D}, d_{1}$ is respectively approximately 0.93 and 0.8 times the stability threshold (as in Figs. 3A and B). Most of the differences between Fig. $3 \mathrm{C}$ and $\mathrm{D}$ and the corresponding Figs. $3 \mathrm{~A}$ and $\mathrm{B}$ are the result of the decrease in $a_{1}$ rather than $a_{2}$; comparable figures for $a_{1}=1$ and $a_{2}=5$ are very similar to Figs. $3 \mathrm{C}$ and D. The lower value of $a_{1}$ eliminates most of the chaotic dynamics. When $d_{1}$ is $93 \%$ of its threshold (Fig. 3C), there is a very narrow band of chaos, similar in form to asynchronous cycles, at the boundary between exclusion of the nonlinear predator and coexistence. When $d_{1}$ is $80 \%$ of its threshold and the $a_{i}$ are relatively small (Fig. $3 \mathrm{D})$, there is a narrow band of chaotic dynamics at intermediate values of $d_{2}$. In $3 \mathrm{C}$, the majority of the possible $\left\{a_{2}, d_{2}\right\}$ parameter combinations produce asynchronous cycles. This would also be true of Fig. 3D if we used a correlation coefficient of 0.3 as the dividing line between synchronous and asynchronous. Some of the panels in Fig. 3 appear to be characterized by qualitatively different types of dynamics occurring as roughly parallel bands in $\left(a_{2}, d_{2}\right)$ space. However, the ranges of qualitatively different types of dynamics does change with a sufficiently large proportional change in both $a_{2}$ and $d_{2}$, as is shown in Fig. 3E, where both $a_{2}$ and $d_{2}$ have been reduced from their values in Fig. 3B so that $d_{2}<0.5$. At very low demographic rates of the linear predator $\left(a_{2}\right.$ and $\left.d_{2}\right)$, synchronous cycles are the predominant dynamics when coexistence occurs. Values of $a_{2}$ and $d_{2}$ much larger than 5 (results not shown) tend to make some of the chaotic dynamics more nearly periodic; here there are many cases where dynamics are difficult to classify as chaotic or as asynchronous cycles because of the small magnitude of the maximum Lyapunov exponent.

We have not illustrated cases where $d_{1}$ is considerably below the value at which the system becomes stable. Three of our initial sets of parameters assumed that $d_{1}$ is 0.4 times the stability threshold value. For all of these, simple synchronous cycles occur across all of the parameter space where coexistence occurs. This predominance of synchronous cycles characterized all parameter combinations of $a_{1}$ and $d_{1}$ where $d_{1}$ was substantially below the stability threshold. We also do not illustrate cases with very large values of both $a_{i}$ and $d_{i}$. These parameter ranges generally produce more

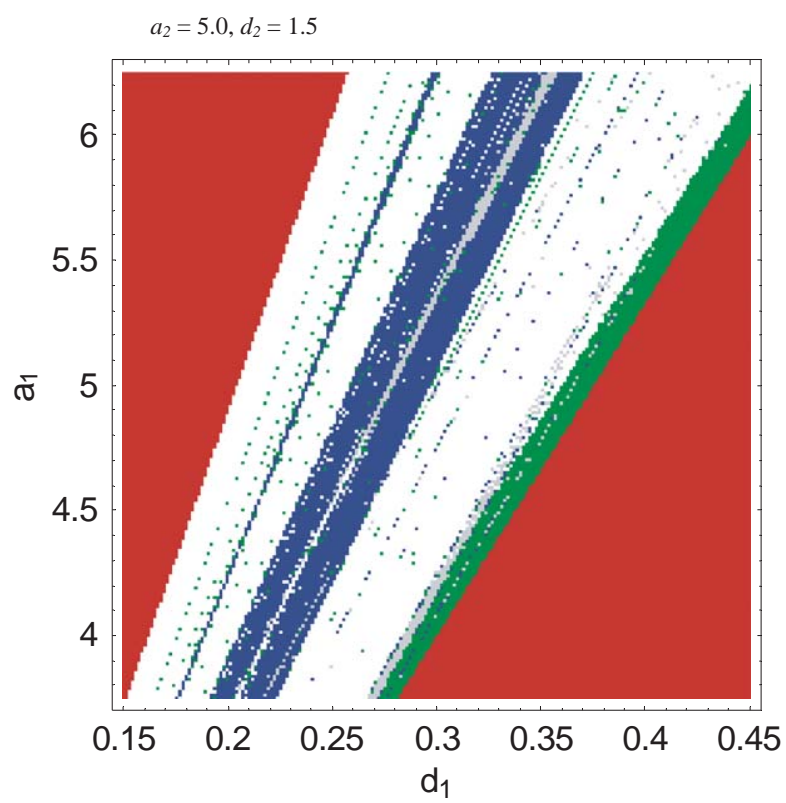

Fig. 4. Dynamics across a grid of $\left\{a_{1}, d_{1}\right\}$ values for a representative value of $a_{2}$ and $d_{2}\left(a_{2}=5 ; d_{2}=1.5\right.$ with $\left.b=10\right)$. Methods and colorcoding are identical to those for Fig. 3.

periodic and fewer chaotic dynamics than do those illustrated here, but they also frequently produce extremely low minimum predator densities.

Fig. 4 presents a different cross-section of parameter space, where $a_{2}$ and $d_{2}$ are fixed, and dynamic behaviors across a range of $a_{1}$ and $d_{1}$ values are illustrated. The region of asynchronous or chaotic dynamics is a minority of the range of parameters where the two predators can coexist in this figure; nevertheless, this region constitutes a significant fraction of parameter space. That fraction decreases when $a_{1}$ is decreased. In general, the results from Figs. 3 and 4 support the qualitative comments made above regarding the occurrence of the three classes of dynamical behaviors across the range of parameter values that permit coexistence.

\section{Responses of mean predator densities to altered demographic parameters}

In this section we examine how the mean densities of the two competitors are affected by changes in demographic rates. One goal of this exercise is to clarify how competition in this model differs from competition in models with stable, equilibrial coexistence arising from classical niche partitioning. There are a number of properties common to models of the latter type, as described in the Introduction. As shown below, the responses of predator densities to changes in demographic rates are very different in the ArmstrongMcGehee model. We again largely confine our numerical results to cases in which $b=10$, and assume a small 


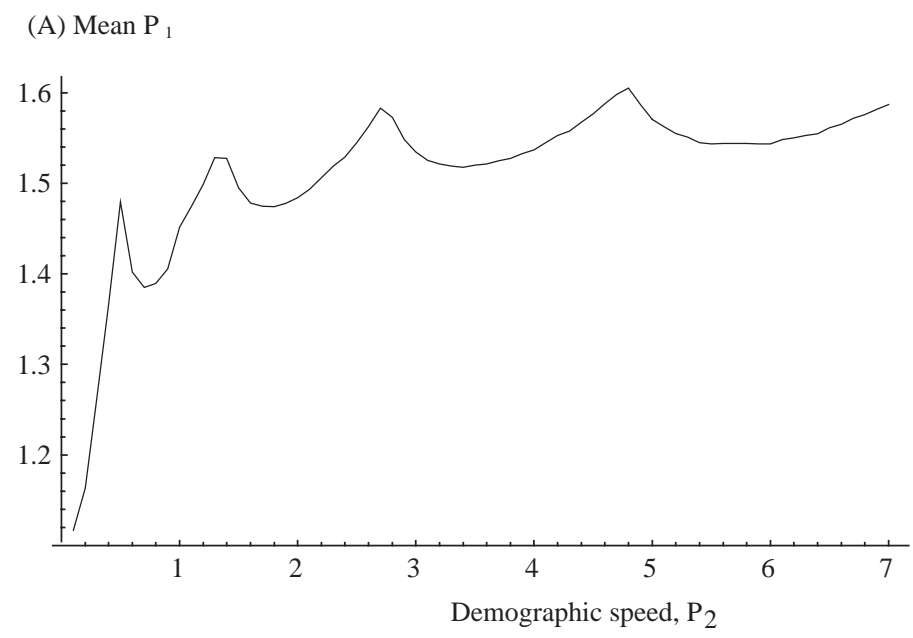

(B) Mean $\mathrm{P}_{2}$

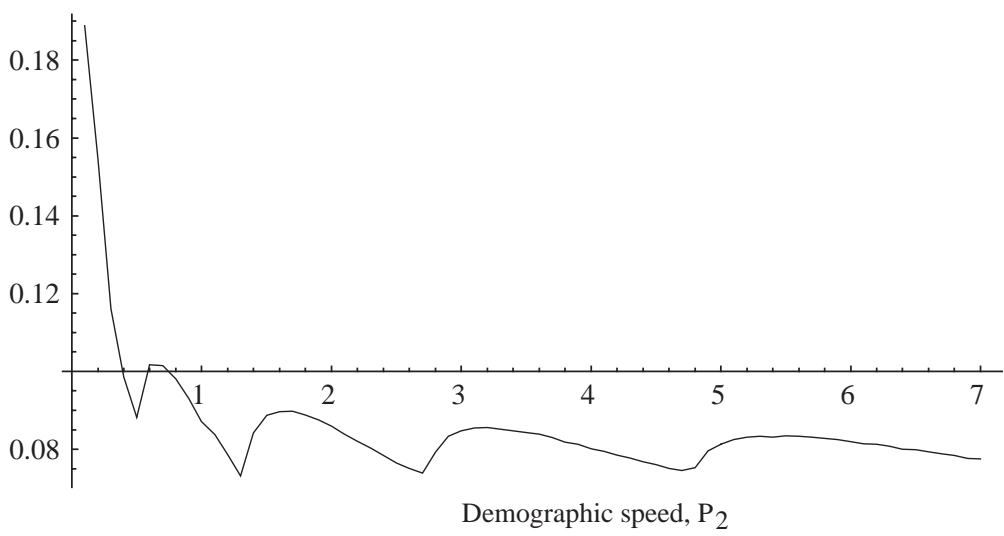

Fig. 5. Population densities of the two predators as a function of the demographic rates of species 2, the linear predator. The baseline parameters from Eqs. (1) are $C_{1}=C_{2}=1, h_{1}=10, B_{1}=B_{2}=1, D_{1}=0.06, D_{2}=0.4, r=1, K=1$. 'Demographic speed' means the scaling factor multiplying both $B_{2}$ and $D_{2}$. Values smaller than 1 result in synchronized limit cycle dynamics, while values equal to or larger than 1 all produce chaotic dynamics.

amount of prey immigration. We measure interspecific effects by calculating changes in mean densities, because this is the best single measure of the impact of the affected species on other species in the community (Abrams and Roth, 1994a, b; Abrams et al., 1998), and is the measure used in virtually all field studies that have quantified interspecific interactions. Clearly changes in the pattern of variation can also be important for both ecological and evolutionary questions, but these will not be pursued here. Mean densities in chaotic systems are influenced by the length of the time over which the mean is calculated, but the $10^{4}$ time unit interval used here produced a very close ( $\ll 1 \%$ difference) approximation of the mean for much longer periods for most chaotic parameter sets (longer time intervals were used when the period of the fluctuation was very long, as in Fig. 1B).

We begin by considering equal proportional changes in both demographic rate parameters, $B_{i}$ and $D_{i}$ for one of the two competitors in Eq. (1). This is equivalent to proportional changes in $a_{i}$ and $d_{i}$ in Eq. (2), but the change in $B_{i}$ also changes the scaling of the density of predator $i$. Thus we will use the unscaled equations to examine the effects of changes in demographic rates on population densities. Such changes are analogous to changes in intrinsic growth rates, $r$, in the two-species Lotka-Volterra model, in that model they have no impact on the ultimate densities of either species, and only determine the speed of approach to equilibrium. An example of the mean densities of both competitors in the Armstrong-McGehee model is shown as a function of the demographic rates of the linear predator species in Fig. 5. In general, slower dynamics for this species imply simpler dynamics of the entire system. At the lowest demographic rates shown, the dynamics are simple synchronized cycles, and the mean density of species 2 decreases rapidly (and that of species 1 increases rapidly) as the demographic rates of species 2 are increased. Cycles become more complicated and asynchronous as the demographic scaling factor increases above unity. The complicated multimodal 
(A) Mean $\mathrm{P}_{1}$

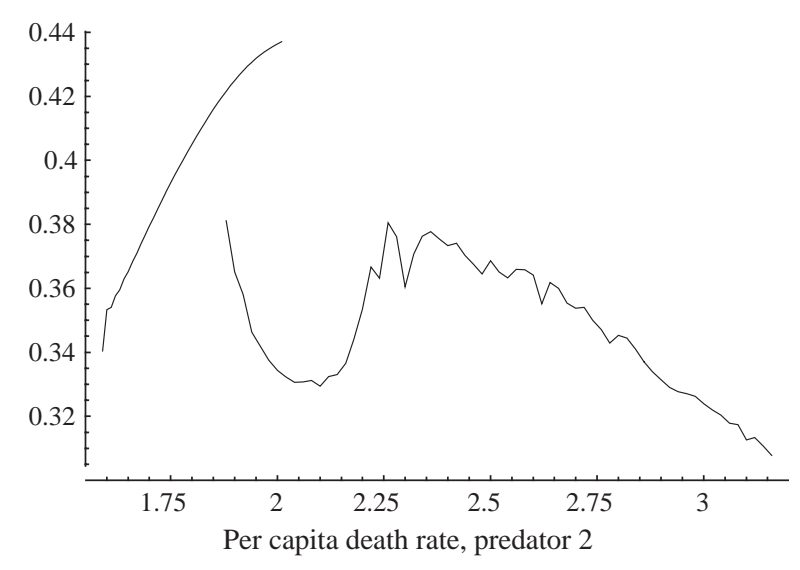

(B) Mean $\mathrm{P}_{2}$

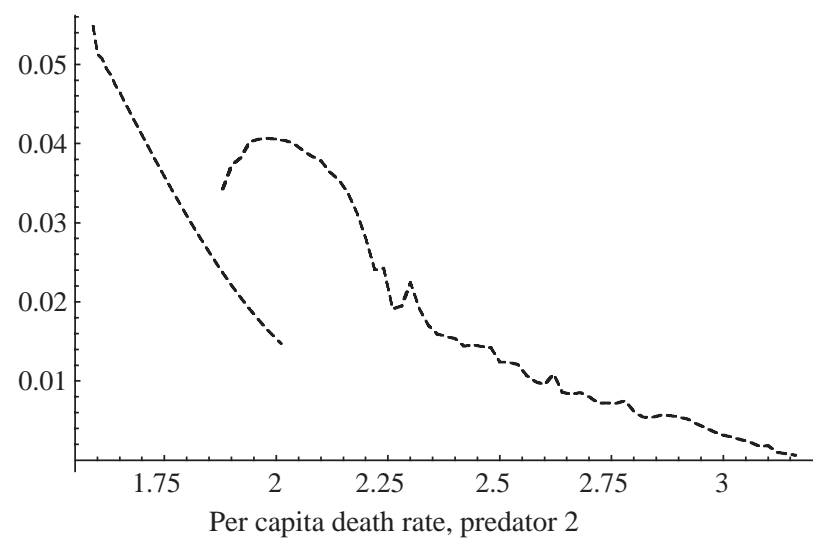

Fig. 6. Mean population densities of both predators in Eq. (2) as a function of the per capita mortality rate of the linear predator, species 2. The parameters for this figure are $b=10, a_{1}=a_{2}=5$, and $d_{1}=$ 0.38 . The instantaneous immigration rate of prey is 0.0001 . This range of $d_{2}$ is characterized by many transitions between chaotic and periodic dynamics.

pattern of mean densities shown in the figure is the consequence of changes in the complexity of the asynchronous cycles with increasing demographic speed. In general, qualitative changes in the nature of the population fluctuations as demographic rates shift will cause significant changes in the mean densities of the two predators for most parameter values. Fig. 3 shows that such qualitative shifts are likely to occur frequently with proportional changes in the two demographic rate parameters of one or both species. Proportional changes in both $a_{1}$ and $d_{1}$ are the only difference between Figs. $3 \mathrm{~B}$ and D, and between Figs. 3A and C; in these cases, slower dynamics of the nonlinear species greatly decrease on the range of parameters that produce chaos, and increase the range of parameters leading to asynchronous cycles. Faster dynamics of predator 1 have the opposite effects. Large proportional decreases in $a_{2}$ and $d_{2}$ cause asynchronous or chaotic dynamics to shift to synchronous limit cycles (Fig. 3E), accompanied by significant changes in mean predator densities.
Mortality rates experience a great deal of natural variation, and can often be manipulated in field or laboratory experiments. Increased mortality of either competitor in the 2-species Lotka-Volterra model decreases its density and increases the competitor's density. In the Armstrong-McGehee model the consequences of altered per capita mortality rates for mean population densities are strongly influenced by the resultant qualitative changes in system dynamics. Figs. 6 and 7 show two examples of the changes in mean population densities that occur with increases in the death rate of one of the two predators in Eq. (2). In both figures, the system changes through all three of the qualitative types of dynamics as the mortality rate of one of the species is changed. Significant features of the results that differ from standard equilibrium models of competition are:

(1) The mean density of a predator may increase with its own death rate. This is true of the linear predator species (2) over a limited range of mortalities, as shown in Fig. 6. It is true of the nonlinear predator (1) over most of the range of death rates that allow coexistence, as shown in Fig. 7.

(2) The mean density of a predator may increase with decreases in the death rate of its competitor. This is true of species 1 over roughly the upper half of the
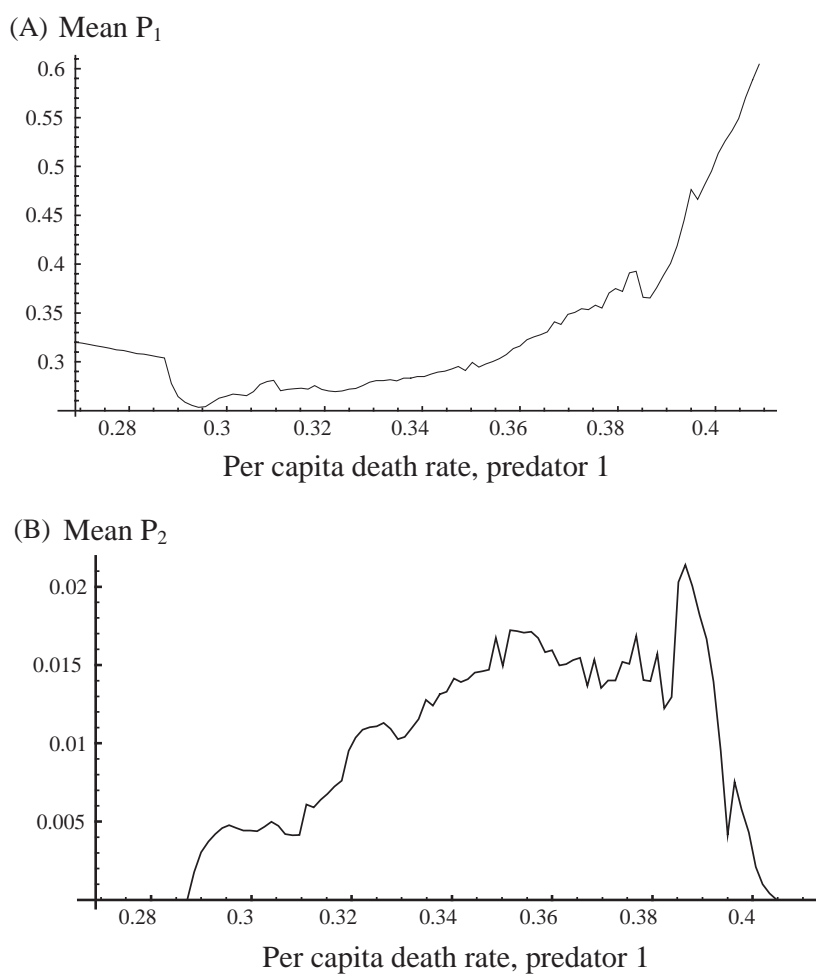

Fig. 7. Mean population densities of both predators in Eq. (2) as a function of the per capita mortality rate of the nonlinear predator, species 1. The parameter values are the same as those in Fig. 6 with the exception that $d_{2}=2.4$. 
range of death rates of its competitor that permit coexistence (Fig. 6). It should also be noted that the mean density of species 1 when alone is approximately given by its density at the right-hand side of Fig. $6\left(d_{2}=3.2\right)$; the graph shows that the presence of species 2 increases the mean density of species 1 for all of the possible range of mortality rates of species 2 that allow coexistence. In Fig. 7, an increase in species 1 mortality decreases the density of species 2 over the upper part of the range of per capita mortality rates of species 1 that allow coexistence. In fact, raising the death rate of species 1 actually results in exclusion of species 2 in this example, because the system becomes stable at $d_{1}=$ 0.409 . However, a small additional increase in $d_{1}$ to 0.42 in Fig. 7 would reverse the outcome, and species 2 would then exclude 1 . This example is also interesting because both species increase in response to increased mortality of species 1 over most of the range of potential death rates.

(3) When there are alternative attractors, the mean population densities of a given predator often respond in opposite directions to changes in one of the two per capita death rates in the two attractors (as is true in Fig. 6 over most of the range of mortality rates of predator 2 where alternative attractors occur).

(4) A change in the mortality rate of one predator that alters the number of predator species present often results in a discontinuous change in the mean densities of both predators. Although not shown in Fig. 6, there are large and abrupt changes in the densities of both predators when the death rate of the linear predator drops to a level where the nonlinear predator is excluded. When $d_{2}$ decreases from 1.59 to 1.58 in that example, the density of predator 2 increases from 0.053 to 0.1364 , while the density of predator 1 drops from 0.348 to zero. These discontinuous changes in mean densities at the lower end of the range of $d_{2}$ that allows coexistence appear to occur for all of parameter space yielding coexistence for Eq. (2). In all cases, the linear predator attains a much higher abundance when the nonlinear predator is excluded.

Many of these features of the responses of densities to mortality rates can be understood heuristically by considering the consequences of altered mortality for the amplitude of predator-prey cycles. Higher predator mortality decreases the amplitude of limit cycles in the nonlinear subsystem composed of the predator and prey. Higher mortality also results in a relatively slow increase in the predator's mean density in this subsystem (Abrams and Roth, 1994a; Abrams et al., 1997). The latter effect arises from the fact that mortality actually increases the predator's density at the equilibrium point, but the large amplitude cycles reduce the mean growth rate, and therefore density, of the predator. The presence of the linear predator dampens these oscillations, and this effect alone would increase the mean density of the nonlinear predator above its mean density in isolation. This is why all of the cases where predator 2 is present in Fig. 6 have a higher mean density of predator 1 than when predator 2 is absent (i.e. at $d_{2}=3.2$ ). The increase in the mean densities of both predators with increased mortality of predator 1 (true of most of the range of $d_{1}$ shown in Fig. 7) is a consequence of smaller amplitude cycles. Similarly, the decrease in the density of the nonlinear predator (species 1) with increasing $d_{2}$ in Fig. 6 is a consequence of the weakening effect of the linear species in damping the prey oscillations driven by the nonlinear predator.

The discontinuities in mean predator density that occur (e.g., just beyond the left-hand side of Fig. 6, and at the right-hand side of Fig. 7) result from major changes in the amplitude of cycles at those points, with concomitant change in mean resource density. In Fig. 7, species 2 is excluded when $d_{1}$ reaches its stability threshold. Which species is excluded when $d_{1}$ is increased above the stability threshold given by Eq. (3) depends on the prey requirement for zero growth in the linear species. Exclusion of the linear species 2 is more likely if its own prey requirement is relatively high. The exact requirement for exclusion of species 2 at high $d_{1}$ as the result of stabilization of the system from inequality (3) is $d_{2} / a_{2}>(b-1) /(2 b)$.

If the per capita mortality rate of species 1 is sufficiently low relative to the stability threshold, complicated and/or chaotic dynamics are typically replaced by simple synchronous cycles; changes in mean densities with altered death rates are in the direction expected based on the intuitive notion that negative effects (i.e., higher mortality) inflicted on one competitor increase the mean density of its competitors.

Other parameters that could change in this system include immigration rates and prey carrying capacity. Prey immigration tends to be stabilizing, and so generally increases dominance of the nonlinear predator. Increasing $K$ is equivalent to proportional increases in both $a_{i}$ and in $b$; this environmental change increases the amplitude of cycles, and generally increases the abundance of the species with the linear functional response, which benefits more from periods of high prey density.

\section{Dynamics and responses to mortality rates in related models}

The specific system illustrated in Armstrong and McGehee (1980) (Eq. (1) above) is only one exemplar of a mechanism that arises in a broad range of models. It is 
important to gain insight into how the above results change when model components are changed, particularly with respect to changes that increase the probability of coexistence of predator species. There are too many different conceivable modifications of Eq. (1) to permit as thorough an exploration as has been carried out above. In this section we present preliminary results regarding the following modifications: (1) different prey growth functions; (2) an accelerating rather than a linear functional response for the stable predator; (3) immigration of one or both predators; (4) a type-2 functional response for both predators; (5) more than one resource. Many other mechanisms producing temporal variation in densities may also alter the responses of competing species to environmental change (e.g., Chesson, 1986, 1994, 2000), but these will not be discussed here.

A flexible description of prey density-dependence is provided by the 'theta-logistic' (Gilpin and Ayala, 1973) model, where $d N / d t=r N\left[1-(N / K)^{\theta}\right]$. We have carried out a number of simulations where $\theta$ is either significantly larger or smaller than 1 . Moderate changes in $\theta$ do not change the general conclusions reached above. Larger values of $\theta$ increase the range of relative mortalities that allow coexistence (Abrams and Holt, 2002). The dynamics observed across a range of death rates resemble the case of $\theta=1$, with the same three general classes of dynamics identified above. Synchronous cycles were observed in all systems when $d_{1}$ is sufficiently lower than its threshold value for stability, regardless of $d_{2}$. The responses of mean densities to altered per capita mortality rates were, however, affected by the form of density dependence. Fig. 8 shows the responses of the mean predator densities to altered per capita mortality rates of the linear predator for two systems that differ only in their density dependent exponent, $\theta$. The major difference between these and comparable models with $\theta=1$ (logistic growth), is that the abrupt jump in mean density when $d_{2}$ is just large enough to allow the nonlinear predator to exist does not occur at large values of $\theta$. This jump is more pronounced for small values of $\theta$ than for the logistic model.

Abrams and Holt (2002) showed that, if the species with a linear functional response was replaced by one with an accelerating functional response, the range of mortality rates or other parameters allowing coexistence (the 'coexistence bandwidth'; Armstrong, 1976) was expanded. We carried out some numerical analyses of such a model to determine the range of dynamics and responses to mortality rates. The accelerating functional response strengthens the stabilizing impact of the stable predator species on the 3 -species system. As a result, there was an increase in the range of parameter space within which the stable predator could control the prey fluctuations and produce asynchronous cycles, particularly when the death rate of the unstable predator was
(A) $\theta=0.5$

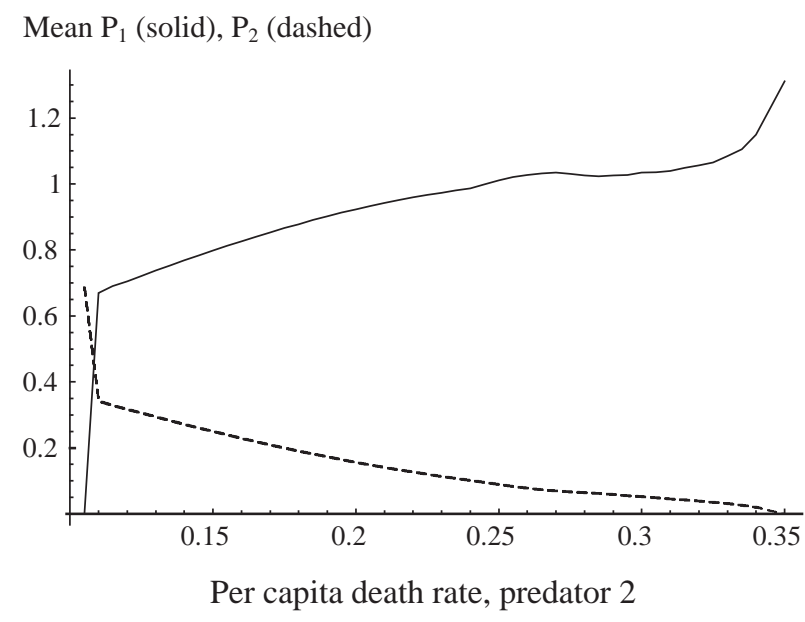

(B) $\theta=4$

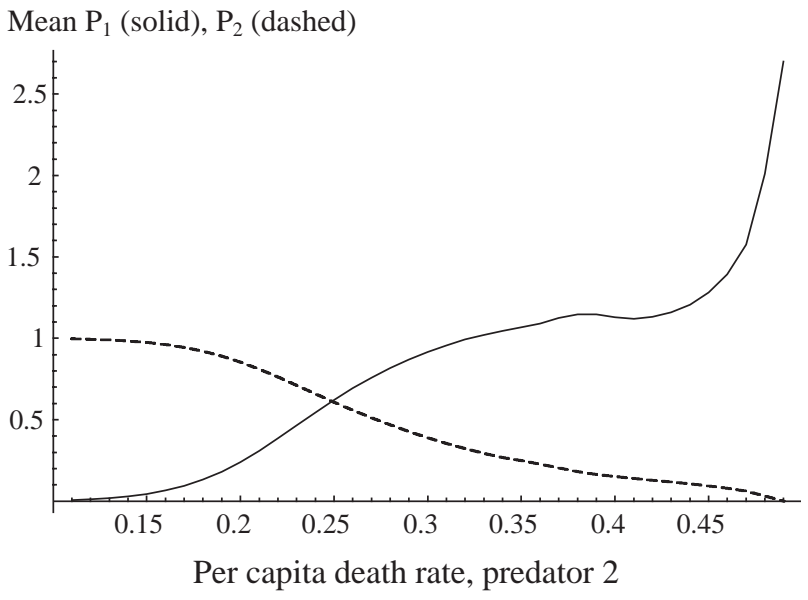

Fig. 8. The mean densities of the two predators as functions of the per capita mortality rate of the linear predator species for two models in which the prey has $\theta$-logistic growth, with $\theta=0.5$ in the top panel and $\theta=4$ in the bottom panel. Otherwise the model is identical to Eq. (2) with the following common parameters for both panels: $b=10, a_{1}=$ $a_{2}=1, d_{1}=0.05$. There is no prey immigration.

relatively close to its stability threshold value. Chaotic dynamics, when they occurred, were generally shifted to relatively low values of the death rate of the stable predator, within the range allowing coexistence.

Predator immigration may permit the local coexistence of two or more predator species at significant densities over a broader range of parameters than for the models described above, with their closed predator populations. We have explored the dynamics of a model consisting of Eq. (1) with constant immigration of all species. Low levels of predator immigration do not alter the general features reported above: the same three categories of dynamics were observed for approximately the same ranges of parameter values. Immigration of predator 2 does eliminate some cases of extremely low densities of that predator. High immigration of the nonlinear predator reduces the amplitude of its cycles 
with the prey, and therefore reduces the range of parameters over which there can be persistence of a linear predator that does not also have immigration. The responses of mean population densities of both predators to an altered immigration rate of one of the predators is typically very nonlinear. For example, increased immigration of predator 1 in a system whose other parameters are those given in Fig. 7, often causes a decrease in the mean density of predator 1, until immigration is nearly sufficient to cause extinction of predator 2 . The mean density of predator 2 changes in a highly nonlinear manner with increasing immigration of predator 1 as a consequence of the nonlinear effect of immigration on cycle amplitude. Moreover, relatively small immigration rates can allow coexistence of three of more predator species at significant densities, something which requires very delicate balancing of parameters when there is no immigration (Chesson, 1994; Abrams and Holt, 2002). Thus, in a metapopulation context, there may be local diversities much greater than two predator species, given predator-prey cycles and differences in the linearity of predator responses to prey density.

We have also examined models in which each of the two predators has a saturating functional response, but differ in the half-saturation constant ( $b$ in Eq. (2)). This system has previously been studied numerically by Hsu and Hubbell (1978) and Abrams and Holt (2002). As pointed out in Abrams and Holt (2002), coexistence requires very precise balancing of parameters when both species have substantial half-saturation constants. Thus, coexistence in this more general model is likely to involve significant differences in handling time, such that the functional response of one predator is close to linearity, while the other has a half-saturation constant (b) much greater than unity. These conditions lead to dynamics very similar to those that characterize Eq. (1).

There are many other possible models of competing predators that differ in the linearity of their responses, and undergo predator-prey cycles. For example, Abrams and Holt (2002) consider a case with two prey species, and two predators that differ in their relative consumption rates of the different prey. Such models can exhibit an even wider range of dynamics than do the systems considered here, but our simulations of cases in which there is high similarity between the two predators in their relative prey capture rates displayed a range of dynamics similar to those described for the comparable single-resource Armstrong-McGehee system. Their responses to changes in predator parameter values are also similar to the figures presented here.

Models that add random or seasonal variation in one or more parameters to the basic structure of Eq. (2) have also been studied. These results will be reported in more detail elsewhere (Abrams, under review). In most cases, small levels of variation have relatively small effects on the qualitative form of the dynamics or the responses to parameter values. However, larger amounts of variation can cause major changes in the parameter ranges allowing coexistence as well as in the dynamics of species when they do coexist (Abrams, unpublished manuscript). This is not surprising, given that seasonal variation in prey growth is capable of producing a wide range of complicated dynamics even in predator-prey models otherwise characterized by a stable equilibrium point (Rinaldi and Muratori, 1993; Abrams, 1997b).

\section{Discussion}

The models explored here show that competition between cycling species that differ in the linearity of their responses differs in many ways from the expectation of stable, Lotka-Volterra systems. Smooth changes in parameter values can cause abrupt transitions in the stability of particular attractors of this dynamical system. One can thus see discontinuous changes in mean population densities with continuous changes in mortality. The phenomenon of abrupt changes in dynamical systems with continuous parameter change is common, and has been reviewed for other ecological systems elsewhere (e.g. May, 1977; Scheffer et al., 2001). However, these sorts of abrupt jumps are not a property of familiar two-species models of competition without cycles. Changes in the quantitative or qualitative aspects of population cycling explain the counter-intuitive responses of mean densities to changes in mortality. These include the fact that mortality applied to one predator species can decrease the mean density of its competitor, or even lead to its extinction. Other counterintuitive responses of mean densities to parameter shifts have been noted in other models of interacting populations (Armstrong and McGehee, 1980; Holt, 1983; Abrams and Roth, 1994a, b; Abrams, 1997a, b; Abrams et al., 1997, 1998; Rinaldi and DeFeo, 1999) when there are sustained fluctuations and when per capita growth rates are nonlinear functions of densities. The current model actually presents a methodological problem for some population definitions of competition, because addition of the linear predator often increases the mean density of the nonlinear predator.

Several of the dynamical transitions we have here documented should be useful in identifying cases where the coexistence-promoting mechanism described here may be operating. Bifurcations in dynamics have been useful in identifying the mechanism of population regulation in flour beetles (Dennis et al., 2001) and the mechanism driving predator-prey cycles in a rotifer-alga system (Fussmann et al., 2000). Altering the per capita mortality of a species is often possible in both field and lab settings. If the Armstrong-McGehee mechanism promotes coexistence in a system, our analysis has 
identified some characteristic transitions in the dynamics that occur, particularly when the mortality rate of the stable species is increased. It may also be possible to slow the population dynamics or reduce the preycapture rates of one or both predators in some experimental setting (e.g. Luckinbill, 1973); these manipulations are also expected to lead to characteristic transitions between different types of dynamics.

The three broad classes of dynamics observed for the simple Armstrong-McGehee (1980) model also arise in a variety of related models that share the same basic mechanism of coexistence. The asynchronous type of cycles are particularly significant, because they suggest that short-term observations may often give a highly misleading picture of the long-term behavior of systems of cycling competitors. The period of gradual replacement of the stable predator by the unstable one may last many generations, and give an impression of ultimate competitive exclusion. However, the relative abundances of the two species can eventually be reversed in a very short time span. Although simple limit cycles with positively correlated predator populations occur over the largest area of parameter space, this does not mean that such cycles in fact represent the most common dynamic pattern in natural systems exhibiting this mechanism of coexistence. Systems in which the unstable predator has a death rate close to the stability threshold are more likely to avoid stochastic extinctions than are systems with lower predator mortality rates. Mortality rates near the threshold are in turn associated with a high probability of chaotic dynamics, complex limit cycles, or alternative attractors. Although complex dynamics have been described for nonlinear consumerresource models of competition (Vandermeer, 1993), very little modeling of competition considers the possibility of cycling, let alone the consequences of the changes in cycling that are caused by changes in a parameter of the competing species.

Finally, it should be noted that many natural populations that are harvested are themselves predators in food webs, which are likely to display unstable dynamics. The changes in death rates explored above can be broadly interpreted as changes in harvesting rates. Our results suggest that one could be lulled into a false sense of security because a focal species maintains high average abundance (or even increases) as its harvest increases (see also Abrams, 2002).

\section{Acknowledgments}

An operating grant from the Natural Sciences and Engineering Research Council of Canada and a fellowship from the J.S. Guggenheim Foundation provided financial support to PAA. RDH acknowledges support from the National Science Foundation (USA) and the
University of Florida Foundation. CEB was supported by an Ontario Graduate Scholarship. We are greatly indebted to the National Center for Ecological Analysis and Synthesis for sponsoring the working group on competition theory, which provided the intellectual impetus for this work. NCEAS is supported by NSF (Grant \#DEB-0072909), the University of California, and UC Santa Barbara.

\section{References}

Abrams, P.A., 1997a. Evolutionary responses of foraging-related traits in unstable predator-prey systems. Evol. Ecol. 11, 673-686.

Abrams, P.A., 1997b. Variability and adaptive behavior; implications for interactions between stream organisms. J. N. Am. Benth. Soc. $16,358-374$

Abrams, P.A., 2002. Will small population sizes warn us of impending extinctions? Am. Nat. 160, 293-305.

Abrams, P.A., Hill, C., Elmgren, R., 1990. The functional response of the predatory polychaete, Harmothoe sarsi to the amphipod, Pontoporeia affinis. Oikos 59, 261-269.

Abrams, P.A., Holt, R.D., 2002. The impact of consumer-resource cycles on the coexistence of competing consumers. Theor. Popul. Biol. 62, 281-296.

Abrams, P.A., Holt, R.D., Roth, J.D., 1998. Shared predation when populations cycle. Ecology 79, 201-212.

Abrams, P.A., Namba, T., Mimura, M., Roth, J.D., 1997. Comment on Abrams and Roth: the relationship between productivity and population densities in cycling predator-prey systems. Evol. Ecol. 11, 371-373.

Abrams, P.A., Roth, J.D., 1994a. The responses of unstable food chains to enrichment. Evol. Ecol. 8, 150-171.

Abrams, P.A., Roth, J.D., 1994b. The effects of enrichment on threespecies food chains with nonlinear functional responses. Ecology 75, 1118-1130.

Abrams, P.A., Shen, L., 1989. Population dynamics of systems with consumers that maintain a constant ratio of intake rates of two resources. Theor. Popul. Biol. 35, 51-89.

Armstrong, R.A., 1976. Fugitive species: experiments with fungi and some theoretical considerations. Ecology 57, 953-963.

Armstrong, R.A., McGehee, R., 1976a. Coexistence of two competitors on one resource. J. Theor. Biol. 56, 499-502.

Armstrong, R.A., McGehee, R., 1976b. Coexistence of species competing for shared resources. Theor. Popul. Biol. 9, 317-328.

Armstrong, R.A., McGehee, R., 1980. Competitive exclusion. Am. Nat. 115, 151-170.

Case, T.J., 1995. Surprising behavior from a familiar model and implications for competition theory. Am. Nat. 146, 961-966.

Chesson, P., 1986. Environmental variation and the coexistence of species. In: Diamond, J.M., Case, T.J. (Eds.), Community Ecology. Harper and Row, New York, pp. 240-256.

Chesson, P., 1994. Multispecies competition in variable environments. Theor. Popul. Biol. 45, 227-276.

Chesson, P., 2000. Mechanisms of maintenance of species diversity. Ann. Rev. Ecol. Systems 31, 343-366.

Dennis, B., Desharnais, R.A., Cushing, J.M., et al., 2001. Estimating chaos and complex dynamics in an insect population. Ecol. Monogr. 71, 277-303.

Eby, L.A., Rudstam, L.G., Kitchell, J.F., 1995. Predator responses to prey population dynamics-an empirical analysis based on lake trout growth rates. Can. J. Fish. Aquat. Sci. 52, 1564-1571. 
Ellner, S.P., Turchin, P., 1995. Chaos in a noisy world; new methods and evidence from time-series analysis. Am. Nat. 145, 343-375.

Fussmann, G.F., Ellner, S.P., Shertzer, K.W., 2000. Crossing the hopf bifurcation in a live predator-prey system. Science 290, $1358-1360$

Gilpin, M.E., Ayala, F.J., 1973. Global models of growth and competition. Proc. Natl. Acad. Sci. (USA) 70, 3590-3593.

Gross, J.E., Shipley, L.A., Hobbs, N.T., Spalinger, D.E., Wunder, B.A., 1993. Functional response of herbivores in food-concentrated patches: tests of a mechanistic model. Ecology 74, 778-791.

Holt, R.D., 1983. Immigration and the dynamics of peripheral populations. In: Miyata, K., Rhodin, A. (Eds.), Advances in Herpetology and Evolutionary Biology. Harvard University Press, Cambridge, pp. 680-694.

Hsu, S.B, Hubbell, S.P., Waltman, P., 1978. A contribution to the theory of competing predators. Ecol. Monog. 48, 337-349.

Kendall, B.E., Predergast, J., Bjørnstad, O., 1998. The macroecology of population dynamics: taxonomic and biogeographic patterns of population cycles. Ecol. Lett. 1, 160-164.

Koch, A.L., 1974. Competitive coexistence of two predators utilizing the same prey under constant environmental conditions. J. Theor. Biol. 44, 387-395.

Luckinbill, L.S., 1973. Coexistence in laboratory populations of Paramecium aurelia and its predator Didinium nasutum. Ecology $54,1320-1327$.

May, R.M., 1977. Thresholds and breakpoints in ecosystems with a multiplicity of stable states. Nature 269, 471-477.
McGehee, R., Armstrong, R.A., 1977. Some mathematical problems concerning the ecological principle of competitive exclusion. J. Differential Equations 29, 214-234.

Messier, F., 1994. Ungulate population models with predation: a case study with the North American Moose. Ecology 75, 478-488.

Nusse, H.E., Yorke, J.A., 1998. Dynamics: Numerical Explorations, 2nd Edition. Springer, New York.

Press, W.H., Teukolsky, S.A., Vetterling, W.T., Flannery, B.P., 1992. Numerical Recipes in C. Cambridge University Press, Cambridge.

Rinaldi, S., DeFeo, O., 1999. Top-predator abundance and chaos in tritrophic food chains. Ecol. Lett. 2, 6-10.

Rinaldi, S., Muratori, S., 1993. Conditioned chaos in seasonally perburbed predator-prey models. Ecol. Model 69, 79-97.

Rosenzweig, M.L., MacArthur, R.H., 1963. Graphical representation and stability conditions of predator-prey interactions. Am. Nat. 97, 209-223.

Ruesink, J.L., 1997. Variation in per capita interaction strength: thresholds due to nonlinear dynamics and nonequilibrium conditions. Proc. Natl. Acad. Sci. (USA) 95, 6843-6847.

Scheffer, M., Carpenter, S., Foley, J.A., Folke, C., Walker, B., 2001. Catastrophic shifts in ecosystems. Nature 413, 591-596.

Vandermeer, J.H., 1993. Loose coupling of predator-prey cycles: entrainment, chaos, and intermittency in the classic MacArthur consumer-resource equations. Am. Nat. 141, 687-716.

Wolf, A., Swift, J.B., Swinney, H.L., Vastano, J.A., 1985. Determining Lyapunov exponents from a time series. Physica D 16, 285-317.

Wolfram Research, 1999. Mathematica 4.0. Wolfram Research, Champaign, IL. 